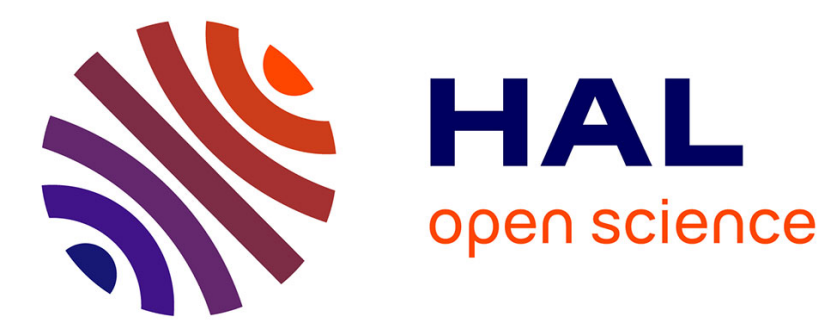

\title{
Des scénarios pour explorer les modèles d'affaires
}

Marie-Laurence Caron-Fasan, Valérie Chanal

\section{To cite this version:}

Marie-Laurence Caron-Fasan, Valérie Chanal. Des scénarios pour explorer les modèles d'affaires. 2008. halshs-00370756

\section{HAL Id: halshs-00370756 \\ https://shs.hal.science/halshs-00370756}

Submitted on 25 Mar 2009

HAL is a multi-disciplinary open access archive for the deposit and dissemination of scientific research documents, whether they are published or not. The documents may come from teaching and research institutions in France or abroad, or from public or private research centers.
L'archive ouverte pluridisciplinaire HAL, est destinée au dépôt et à la diffusion de documents scientifiques de niveau recherche, publiés ou non, émanant des établissements d'enseignement et de recherche français ou étrangers, des laboratoires publics ou privés. 


\title{
Des scénarios pour explorer les modèles d'affaire
}

\section{Publié dans Expansion Management Review, $n^{\circ}$ 128, mars 2008}

\author{
Marie-Laurence CARON-FASAN, Maître de Conférences \\ Université de Grenoble \\ Laboratoire CERAG \\ Equipe Uman lab
}

\author{
Valérie CHANAL, Professeur des Universités \\ Université de Grenoble \\ Laboratoire PACTE \\ Equipe Uman lab
}

Un enjeu majeur des projets d'innovation technologique réside dans la capacité des entreprises à imaginer suffisamment tôt leur potentiel de valeur, et ceci dans un contexte de forte incertitude lié tant à la technologie innovante qu'à un marché parfois inexistant.

Cette question se pose avec la même acuité pour les entreprises déjà établies qui mènent des projets de R\&D et pour les entreprises qui se créent autour d'une innovation technologique. En effet, dans l'un ou l'autre des cas, les porteurs de projet d'innovation technologique ont à présenter à différentes parties prenantes le potentiel de création de valeur de leur innovation. S'ils ne sont pas en mesure d'argumenter sur un modèle de création de valeur, ils n'obtiendront pas des parties prenantes les ressources, en particulier financières, qui leur permettront de mener à bien leur projet. Par ailleurs, il leur est également indispensable en phase de conception, d'anticiper les cibles de marchés visés, les usages de la technologie, et la valeur apportée par la solution au client. Ces connaissances conditionnent en effet des choix et arbitrages importants comme la définition du coût objectif, l'arbitrage entre la simplicité et la performance, le design; des choix qui doivent intervenir en amont du processus d'innovation. Enfin, il est important que les porteurs de projet puissent élaborer des hypothèses sur le réseau de valeur qui va délivrer la nouvelle offre au client, afin de préparer la négociation de partenariats de R\&D ou encore la recherche d'un réseau de distribution. 
La démarche présentée dans cet article part de l'idée que les innovations technologiques réussies s'accompagnent souvent d'une innovation dans les business models. Des auteurs comme Chesbrough et Rosenbloom ${ }^{1}$ ont analysé les succès et les échecs d'entreprises créées à partir de technologies développées au sein du laboratoire de R\&D de Xerox (comme 3COM ou ADOBE). Ils ont montré que les innovations technologiques réussies se sont appuyées sur de nouveaux business models, différents du business model de Xerox, basé sur une technologie propriétaire et une chaîne de valeur intégrée. Cette étude vient appuyer l'idée que ces innovations n'auraient pu réussir au sein de Xerox, en raison de phénomènes de rigidité dans les business models, phénomènes appelés aussi en stratégie « dépendance de sentier ».

Les analyses de ces auteurs permettent de conclure à la nécessité d'aider les managers de $\mathrm{R} \& \mathrm{D}$ ou les porteurs de projets d'innovation à intégrer dans leur réflexion le modèle permettant de créer et de capturer de la valeur à partir de la technologie. Ils considèrent que l'on doit apprendre à faire de l'expérimentation sur les business models, de la même façon que l'on en fait sur les technologies, ce qu'ils appellent du « prototypage stratégique ».

Dans cet article, nous présentons une méthode d'exploration de nouveaux business models autour d'une innovation technologique. L'objectif de cette méthode est d'aider les responsables de projets d'innovation technologique à mieux appréhender les conditions de création de valeur à partir de la technologie et à imaginer différentes alternatives de business model permettant d'amener la nouvelle technologie au client. Nous illustrons ensuite cette démarche autour d'un cas d'innovation technologique dans le domaine des télécommunications.

\section{Partie 1 : Explorer de nouveaux business models : une méthode de scénarios}

Le concept de business model, largement utilisé dans les milieux professionnels depuis le développement d'entreprises sur Internet, commence seulement à être défini de façon précise dans la littérature en management ${ }^{2}$. Les différentes définitions articulent les éléments suivants : l'architecture de l'offre et des ressources mises en œuvre, la proposition de valeur pour le client, la position de l'entreprise dans le réseau de valeur, le modèle de revenus. Autrement dit, définir un business model consiste à se poser la question du modèle

\footnotetext{
${ }^{1}$ Chesbrough H. and Rosembloom R. (2002), The role of the business model in capturing value from innovation: evidence from Xerox Corporation's technology spin-off companies, Industrial and Corporate Change, vol 11, $\mathrm{n}^{\circ} 3$, p 529-555

${ }^{2}$ Voir l'article de synthèse de Lecoq X., Demil B. et Warnier V. (2006), Le business model un outil d'analyse stratégique, Expansion Management Review
} 
d'entreprise pour créer de la valeur et de la position de l'entreprise au sein du secteur pour capturer la valeur.

Nous commençons par présenter notre modèle permettant de guider la réflexion des porteurs d'innovation technologique sur un business model possible. Nous montrons ensuite comment nous pouvons accompagner cette réflexion à partir d'une démarche de scénarios.

\subsection{Un modèle générique pour définir son futur business model}

Nous avons établi une première grille à partir d'une revue de littérature sur la notion de business model. Nous avons retenu cinq catégories de questions reprises par quasiment tous les auteurs étudiés ${ }^{3}$ :

- Quelles sont les applications et les clients visés par la technologie?

- Quelle est la proposition de valeur apportée par la technologie?

- Quelles sont les ressources et capacités (internes ou externes) nécessaires pour développer cette proposition de valeur?

- Comment peut s'organiser le réseau de valeur?

- Quelle sera la logique économique de générations de revenus?

Nos recherches ont montré que ces interrogations génériques qui permettent de caractériser schématiquement tout type de business model doivent être complétées par des questions plus fines, spécifiques à la technologie développée. A partir d'un travail d'analyse de la littérature sur les business models innovants dans différents secteurs, ainsi que des tests de notre modèle dans différents contextes, nous avons identifié cinq thèmes supplémentaires qui peuvent être explorés selon le cas :

- Dans quelle mesure peut-on standardiser l'offre (ou au contraire la segmenter) d'un point de vue marketing

- Dans quelle mesure peut-on standardiser l'offre (ou au contraire la segmenter) d'un point de vue technologique (notamment par le développement de standards technologiques ou avec une approche modulaire de la technologie)

- Quel type d'entreprise peut jouer le rôle de firme centrale dans le réseau de valeur ?

- Peut-on s'inspirer de business models existants dans le domaine (par exemple les business models sur le web)?

\footnotetext{
${ }^{3}$ Voelpel S.C., Leibold M. and Tekie E.B. (2004), The Wheel of Business Model Reinvention : How to Reshape your Business Model to leapfrog Competitors, Journal of Change Management, vol. 4, n 3, p. 259-276; Magretta J. (2002), Why Business Models matter, Harvard Business Review, May, p. 86-92.
} 
- Le business model de l'entreprise étudiée est-il amené à évoluer ? selon quelle dynamique?

Ces 10 catégories de questions forment la grille de départ servant à animer des ateliers de réflexion stratégique avec les porteurs de projet afin d'élaborer différents scénarios pour amener la technologie au client.

\subsection{L'élaboration de différents scénarios de business models}

La difficulté de conduire cette démarche avec des porteurs d'innovation technologique réside précisément dans le fait qu'ils n'ont en général pas suffisamment d'éléments pour répondre aux questions présentées ci-dessus. L'objectif de la méthode est alors de les aider à construire des chemins possibles. Pour cela, nous proposons une démarche basée sur les scénarios.

Il existe deux approches différentes dans l'élaboration des scénarios en stratégie. La première procède par une extrapolation du passé et la recherche de tendances lourdes dans l'environnement. Cette approche conduit généralement à la réalisation de scénarios de « continuité » venant renforcer les paradigmes existants. Ils se basent plus sur des techniques de prévisions et tendent à déduire le futur en se basant au mieux sur l'existant, parfois sur le passé. La deuxième approche se base sur l'élaboration de scénarios de « rupture » à travers un processus de construction de sens autour de facteurs d'incertitude. Ces facteurs sont ceux dont on postule qu'ils auront un impact fort (positif ou négatif) sur la stratégie ou le projet. Ces scénarios ont pour objectif de modifier les représentations que les acteurs ont de leur environnement, de mettre en évidence des contradictions et anomalies stratégiques aboutissant à la remise en cause des paradigmes existants et anciens. Plutôt que de chercher à diminuer l'incertitude, ils l'intègrent au contraire comme élément central dans les raisonnements afin d'en tirer avantage. C'est cette perspective que nous adoptons, en concevant les scénarios au sens littéral comme des histoires de futurs possibles. Ils sont susceptibles d'augmenter la créativité, de favoriser l'attention et d'aider les managers à mieux prendre en compte l'incertitude et la complexité de leur environnement.

Ainsi, notre démarche de construction de scénarios se déroule de la façon suivante :

- Au cours d'un atelier stratégique, les porteurs de l'innovation technologique, aidés par les animateurs de la méthode, explorent les différentes catégories de questions (de 1 à 10).

- Pour chacune des questions, les animateurs poussent les porteurs de projet à repérer des alternatives ou des ruptures, et les notent au tableau. L'idée ici est d'amener les porteurs de projet à réfléchir en dehors de leur cadre de référence, en 
imaginant par exemple d'autres segments de marché, d'autres partenaires, un autre modèle de distribution ou encore une autre logique de génération de revenus que celle imaginée a priori. Par exemple, s'ils avaient en tête une logique de génération de revenus basée sur la vente en volumes de produits, on les amène à considérer une proposition de valeur basée sur le service, la vente d'affaires à haute valeur ajoutée, la location etc.

- En regroupant les différentes alternatives proposées sur tous les critères, on peut alors construire différents scénarios (généralement, selon notre expérience, deux ou trois scénarios) dont les différents éléments sont en cohérence. On sera attentif par exemple à la cohérence entre la proposition de valeur pour le client et les ressources mobilisées pour créer et délivrer cette valeur.

\begin{tabular}{|l|l|l|l|}
\hline $\begin{array}{c}\text { Eléments permettant de } \\
\text { caractériser le business } \\
\text { model }\end{array}$ & $\begin{array}{c}\text { Modèle envisagé } \\
\text { par les } \\
\text { innovateurs }\end{array}$ & Alternative 1 & Alternative 2 \\
\hline Applications et clients visés & & & \\
\hline Proposition de valeur & & & \\
\hline Segmentation marketing & & & \\
\hline Ressources nécessaires & & & \\
\hline Standard technologique & & & \\
\hline Type de réseau de valeur & & & \\
\hline $\begin{array}{l}\text { Firme centrale du réseau de } \\
\text { valeur }\end{array}$ & & & \\
\hline Logique économique & & & \\
\hline Business models comparables & & & \\
\hline Evolutions du business model & & & \\
\hline
\end{tabular}

Tableau 1 : Grille schématique de la méthode d'élaboration de scénarios de business models

La démarche se veut délibérément ouverte. Toutes les alternatives sont discutées et analysées, tous les champs possibles sont explorés pour parfois être rejetés, parfois être replacés au 
centre du processus de création de valeur ou parfois être mis en attente de compléments d'informations.

Nous commençons généralement la séance en invitant les participants à définir le plus précisément possible le concept de leur offre. Cette étape peut prendre du temps car les porteurs ont une idée souvent approximative des potentialités réelles offertes par la technologie. Nous abordons ensuite les aspects marketing: identification des domaines d'application, des clients potentiels et formulation de la proposition de valeur pour ces cibles. Il s'agit ensuite d'identifier l'ensemble des ressources stratégiques et des capacités nécessaires pour amener l'offre sur le marché. Cela couvre les ressources et les capacités financières, techniques, logistiques, marketing, etc., aussi bien celles disponibles en interne que celles qu'il faudrait acquérir à l'extérieur. La question de la structure du réseau de valeur est également abordée. Il s'agit de repérer les acteurs qui peuvent prendre part au processus de création de valeur, les acteurs complémentaires et concurrents, ainsi que la répartition de la valeur et les risques éventuels de migration de la valeur vers des acteurs qui auraient moins investi dans le développement de l'offre. Enfin, la démarche aborde la question de la logique de génération de revenus. Elle permet de révéler si le ou les business model imaginés sont suffisamment générateurs de revenus sur le court terme ainsi que sur le moyen terme, et comment le business model identifié est susceptible d'évoluer. Dans le cas des start-ups technologiques en effet, il arrive souvent que les entrepreneurs envisagent une solution de court terme permettant de générer du chiffre d'affaires assez rapidement (la vente de services par exemple) tout en ayant l'idée de faire évoluer leur modèle par la suite, comme la vente de produits.

Généralement, au fur et mesure de l'avancement du travail et de l'exploration systématique des alternatives, on voit émerger deux ou trois scénarios de business models. L'entreprise peut se positionner par exemple comme fournisseur de technologie à des intégrateurs ou OEM, dans ce cas ses compétences seront essentiellement basées sur sa capacité de R\&D. Elle peut, avec la même technologie, envisager un modèle basé sur le développement de produits commercialisés via un réseau de distributeurs, ou encore développer un modèle basé sur la proposition de solutions complètes (produits et services), développées en partenariat avec d'autres acteurs du réseau de valeur. Dans chacun des cas, les clients seront différents, ainsi que les ressources nécessaires et le modèle de génération de revenus.

Ainsi, à l'issue de ce travail, le porteur de projet dispose d'un ou plusieurs scénarios de business model susceptibles d'être mis en œuvre. Cela lui permet de mieux comprendre 
comment sa technologie innovante peut apporter de la valeur pour des clients et selon quel modèle de création de valeur.

Nous avons utilisé cette méthode auprès de plusieurs porteurs de projets de technologie innovante ou responsables de R\&D. Les projets étaient plus ou moins avancés selon les cas et issus de contextes différents :

- une spin-off d'un laboratoire de recherche européen qui vient de rentrer dans un incubateur. Suite à des travaux de recherche, deux porteurs de projets tentent de créer une entreprise sur la base de leur découverte scientifique ;

- une start-up issue d'un grand groupe industriel, qui tente de mettre en place un business model différenciateur, susceptible de générer suffisamment de revenus et qui ne soit pas en concurrence avec leur entreprise d'origine ;

- une start-up, incubée dans une pépinière d'entreprises, qui tente de mettre en place un concept et un business model totalement innovant dans le domaine de la conception de produit high-tech via des communautés virtuelles.

- Un projet de R\&D conduit par un consortium d'industriels et de laboratoires de recherches dans le cadre d'un projet européen autour d'une nouvelle infrastructure de télécommunications.

Dans tous les cas, le déroulement de la démarche a conduit à un enrichissement, voire à une modification du business model tel que les porteurs de projet l'avaient pensé au départ.

Nous développons ci-après les premiers résultats obtenus dans le cadre du projet européen. Les résultats du travail du consortium étant publics, il est en effet plus aisé de communiquer des résultats sur ce cas.

\section{Partie 2 : Une illustration de la méthode d'exploration de business models : le cas e- Sense}

L'intérêt de ce cas est qu'il se base sur une technologie assez générique (une infrastructure de réseaux de capteurs sans fil) pour laquelle la réflexion sur les business models peut paraître prématurée ou en tout cas délicate à mener. Nous montrons cependant que cette réflexion, si elle est articulée avec une démarche d'identification des usages potentiels, peut apporter des éléments intéressants y compris pour le développement de la technologie. Nous commençons par présenter quelques éléments permettant de comprendre le contexte, puis nous expliquons comment le raisonnement stratégique s'est construit, autour d'une application assez simple à 
comprendre, dans le domaine du ski de loisir. Cette application est choisie à titre d'illustration de la démarche.

\subsection{Contexte du projet}

Le projet e-SENSE est un projet de R\&D financé par l'Union Européenne dont l'objectif est de développer des technologies dites «d'intelligence ambiante» pour les systèmes de télécommunication mobiles ${ }^{4}$. L'innovation technologique consiste à développer une infrastructure de télécommunication basée sur des réseaux de capteurs sans fil afin d'apporter des données de contexte à un usager en mobilité. Le projet intègre des développements technologiques (recherche et prototypage) mais également des études de potentiel économique de la technologie sur différents domaines d'application: les services à la personne (dans les loisirs, dans la nutrition), les services aux communautés professionnelles (système de santé sans fil pour les hôpitaux, le suivi des patients à domicile ou la coordination des services d'urgence) et les services industriels (pilotage à distance des marchandises pour les hypermarchés du futur ou le suivi des produits agro-alimentaires). La technologie de eSENSE devrait déboucher sur des innovations disruptives ${ }^{5}$, c'est-à-dire créer de nouveaux marchés ou modifier en profondeur la structure des marchés existants sur la base de nouvelles propositions de valeur.

Nous proposons d'illustrer, dans la suite de cet article, l'utilisation de la méthode dans le cadre de ce projet et plus particulièrement sur le domaine d'application des services à la personne dans le cadre des loisirs, avec un cas d'usage dans le domaine du ski.

\subsection{La vision de la technologie}

La vision technologique de e-SENSE est construite autour d'un concept qui est celui de «l'intelligence ambiante», considérée comme une étape future du développement des réseaux de télécommunications mobiles sans fil après la troisième génération. A ce jour, les technologies qui fournissent des informations sur le contexte aux différents systèmes d'information sont basées sur des éléments disparates, nécessitant des interactions actives de l'utilisateur avec des capteurs spécialisés. La vision autour de e-SENSE consiste à proposer une architecture unique permettant l'interopérabilité de différents types de matériel (capteurs,

\footnotetext{
${ }^{4}$ Les résultats de la recherche menée sur e-SENSE sont publics et accessibles sur le site : www.ist-e-sense.org

5 Christensen C., Anthony S.D. and Roth E.A. (2004), Seeing what's next: Using the theorie of innovation to predict industry change, Harvard Business School Press, Boston
} 
terminaux) et de réseaux, de manière transparente pour l'utilisateur. L'idée est de capturer de manière non intrusive des données de contexte de l'utilisateur (par exemple son niveau de stress, de fatigue, ses gestes etc,) mais aussi des données sur son environnement (par exemple la chaleur, la pollution) ou les produits qu'il utilise, et de traiter ces données afin de fournir des services appropriés, pouvant être délivrés sur différents types de matériels comme des téléphones portables ou des PDA. La création de valeur à travers une offre de services basée sur cette technologie peut impliquer des acteurs aussi divers que des opérateurs de télécommunications, des fabricants de matériel (ordinateurs, téléphones), des fabricants de capteurs, des sociétés d'intégration informatique (SSII) ainsi que de multiples acteurs spécialisés selon les secteurs industriels visés (secteur de la santé, de l'agro-alimentaire, des loisirs etc.).

Les partenaires du consortium européen ont identifié comme potentiellement intéressant le domaine des services à la personne dans le cadre des loisirs et notamment celui du ski, entre autres applications possibles. Un des cas d'usage retenu pour l'étude (parmi une vingtaine de cas dans différents domaines) est le suivant :

- Le skieur est équipé d'un capteur sans fil et d'un système de géo-positionnement au moment où il skie.

- Le système enregistre les trajectoires du skieur sur les pistes ainsi que certains évènements associés à ses trajectoires comme par exemple des sauts ou des chutes.

- L'ensemble des informations sur les trajectoires et évènements associés sont enregistrés dans une base de données.

- Le skieur peut, via la base de données, télécharger sur son téléphone mobile des informations, présentées sous forme de carte, au sujet de ses pratiques de ski (par exemple, ce qu'il a fait dans la journée ou encore ses pratiques de ski sur d'autres journées et dans d'autres stations de ski).

- Chaque information relative à une nouvelle journée de ski, est enregistrée dans la base afin de construire pour chaque skieur un profil personnalisé. On retrouve dans ce profil les pistes sur lesquelles il a skié ainsi que l'enregistrement de différents événements.

- Le skieur peut également télécharger des informations plus générales sur la station et les autres skieurs. Il peut également être informé des pistes ouvertes ou fermées, de l'état de la neige, ou encore des files d'attente à telle ou telle remontée mécanique. Le système peut également fournir des informations sur des services plus traditionnels 
comme localiser ses enfants, ses amis, des membres de sa famille sur le domaine de ski.

Nous exposons ci-après la démarche d'élaboration de scénarios de business models autour de cet exemple.

\subsection{Réalisation de scenarios de business model autour de l'application "ski »}

Sur la base du cas d'usage retenu et compte tenu des intérêts économiques d'un des partenaires du consortium (un opérateur de téléphonie mobile), un travail d'identification de scénarios de business model a d'abord été mené. Nous avons réalisé un premier travail de réflexion stratégique, guidé par notre modèle, avec des responsables du développement de nouveaux business de cet opérateur de téléphonie.

Le concept retenu et sur lequel les scenarios de business model ont été construits est le suivant : «Fournir aux skieurs un service personnalisé d'information multimédia ».

En s'interrogeant sur le premier critère de notre grille (cf. tableau 1) c'est-à-dire sur « qui sont les clients pour ce type de solution ", deux réponses ont été avancées au cours de l'atelier. La première place les skieurs comme «clients finaux». Il s'agit de faire payer directement le service à ceux qui l'utilisent. L'alternative serait de considérer les acteurs de la station de ski (les hôtels, les loueurs de ski, l'office du tourisme de la station, les moniteurs de ski ou encore les managers de la station ou des remontées mécaniques) comme les clients de la solution, à charge pour eux ensuite de délivrer le service au client, sous forme gratuite, payante ou sponsorisée par différents moyens.

A l'issue du travail autour des autres critères de la grille, deux scénarios de business models ont émergé (du point de vue d'un opérateur de téléphonie mobile) : le premier que nous appellerons scénario « skieurs » (vente directe d'un service aux skieurs) et le second scénario « station de ski » (vente d'une solution globale aux stations).

\section{Scénario « Skieurs »}

Les clients sont les skieurs et plus particulièrement les « snow-boarders ». En effet, les tests d'usage de la technologie réalisés dans le cadre du projet ont montré que les communautés de «snow-boarders» sont particulièrement ouvertes à ce type de proposition de nouvelle technologie. La proposition de valeur est multiple. Il s'agit de fournir des informations personnalisées et contextualisées, d'améliorer la sécurité (surtout si les skieurs, ayant pris connaissance des lieux à forte densité de skieurs, vont faire du ski sur des lieux moins fréquentés), de faciliter des pratiques collectives de ski (en proposant de filmer ou de 
photographier les skieurs), de proposer plus de plaisir dans la pratique du ski et enfin de faciliter l'accès aux pratiques de ski.

La figure 1 retrace, pour le scénario «skieurs », l'enchaînement des différents éléments constitutifs du réseau de valeur. Elle montre que l'acteur central (c'est à dire celui qui intègre et vend la solution) est soit la station de ski (et plus explicitement la société d'exploitation des remontées mécaniques ou encore l'Office du tourisme); soit un opérateur de téléphonie mobile (on pourrait ici imaginer des alliances entre opérateurs globaux et locaux). Ces acteurs fournissent directement le service aux «snow-boarders » en leur faisant payer directement le service (par exemple avec la vente du forfait), ou bien en faisant financer le service par des contrats de sponsoring auprès des fabricants d'équipement de ski par exemple.

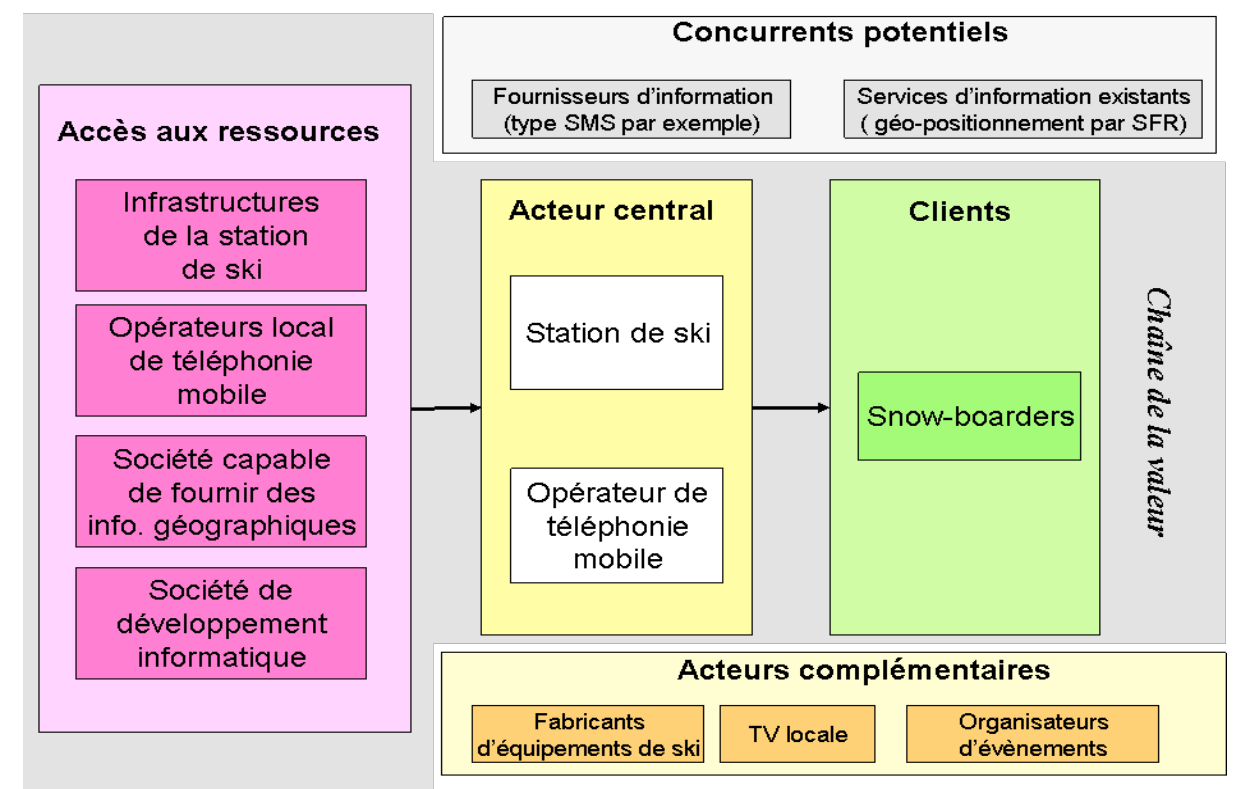

Figure 1 : Réseau de valeur du business model " skieurs»

\section{Scénario « Station de ski»}

Dans ce second scénario, les clients de la solution technologique sont les acteurs de la station de ski. Cela peut être des acteurs institutionnels comme l'office du tourisme ou une société publique d'exploitation des remontées mécaniques, mais aussi des acteurs privés comme les hôtels, les restaurants, les loueurs de matériels ou encore les écoles de ski. La valeur pour la station et ses acteurs est une amélioration de l'attractivité de la station et ainsi la génération d'un flux plus important de skieurs. C'est aussi une amélioration du management de la station en ayant une connaissance plus approfondies des pratiques et aspirations des skieurs.

La figure 2 présente pour le scénario «station de ski» un modèle simplifié du réseau de valeur. L'acteur central dans ce cas serait une société d'ingénierie comme celle qui fournit déjà le système pour la gestion des forfaits main libres dans les stations (basé sur la 
technologie des puces RFID). Le modèle de génération de revenus est ici la vente d'affaires et d'ingénierie, par une société spécialisée à des opérateurs de tourisme comme les stations.

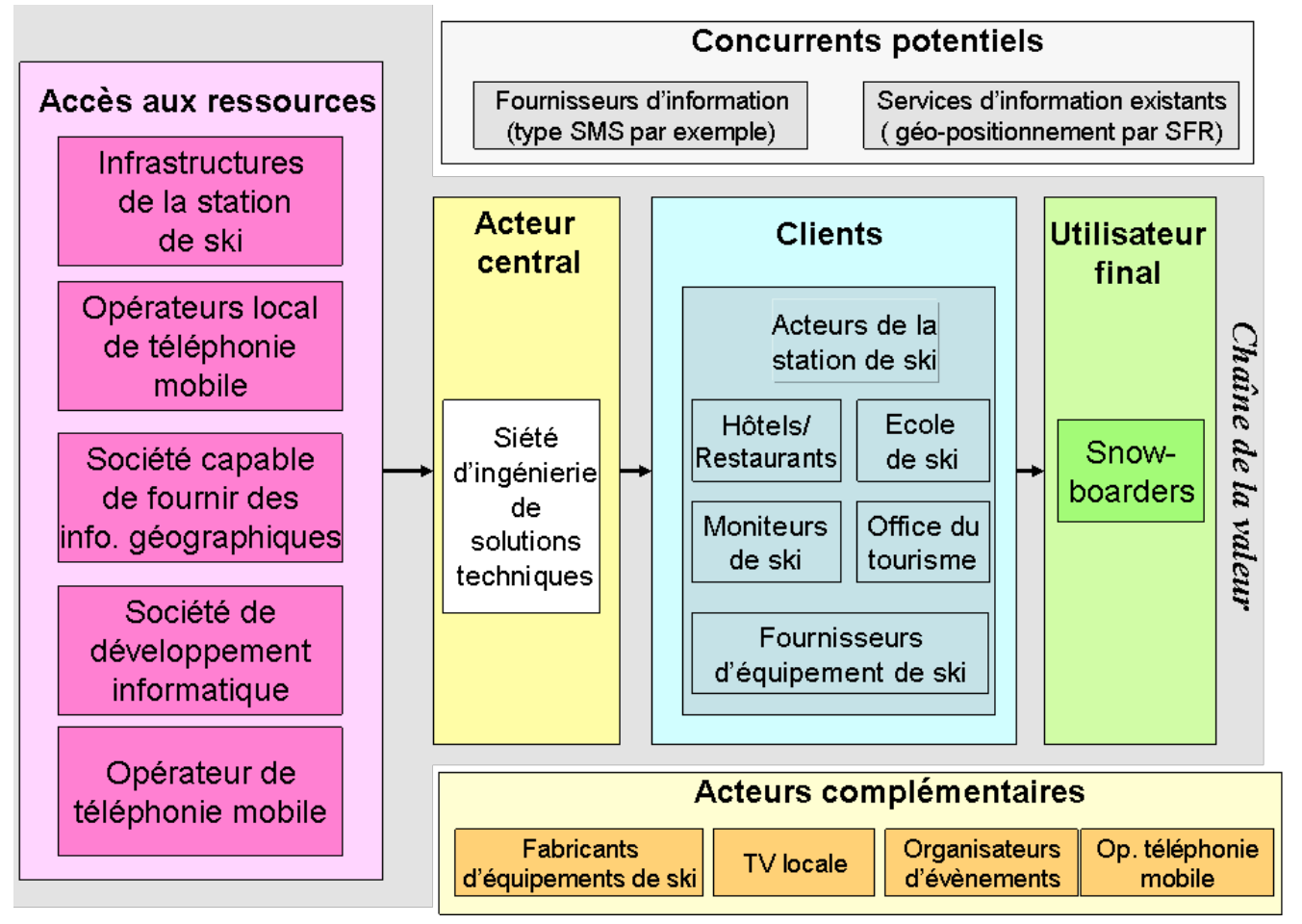

Figure 2 : Réseau de valeur du business model "station de ski»

Dans le scénario «skieurs » une des ressources clé est la ressource marketing qui va permettre d'accéder au marché final; dans le scénario «stations » une des ressources clé est la ressource technologique d'ingénierie de la solution, de la maintenance sur site etc.

Les scénarios retenus peuvent être développés via une standardisation des composants de la solution technique, de manière à rendre réplicable le service sur d'autres stations de ski, dans d'autres contextes et pour d'autres types de skieurs. Une solution plus personnalisée pourrait également être envisagée mais les aspects juridiques relatifs à la collecte et à l'utilisation de données privées semblent constituer un frein important.

Les deux scénarios requièrent de multiples compétences comme des solutions techniques susceptibles de fournir le service, la mise en place d'une politique marketing ainsi qu'une solution de gestion de la relation client (C.R.M.). L'accès à ces différentes compétences suppose la mise en place d'alliances entre différents acteurs: la station de ski et ses infrastructures, un fournisseur d'informations géographiques, des sociétés de développement informatique et des opérateurs locaux de téléphonie mobile.

Ce premier travail exploratoire d'identification de business model sur la base d'un cas d'usage à permis l'identification de deux scenarios de business model possibles. Ce travail a permis 
d'adapter le cas d'usage de départ pour assurer une capture de valeur sur les deux business models identifiés. Ainsi, un concept d'application a clairement émergé, il est représenté dans la figure 3.

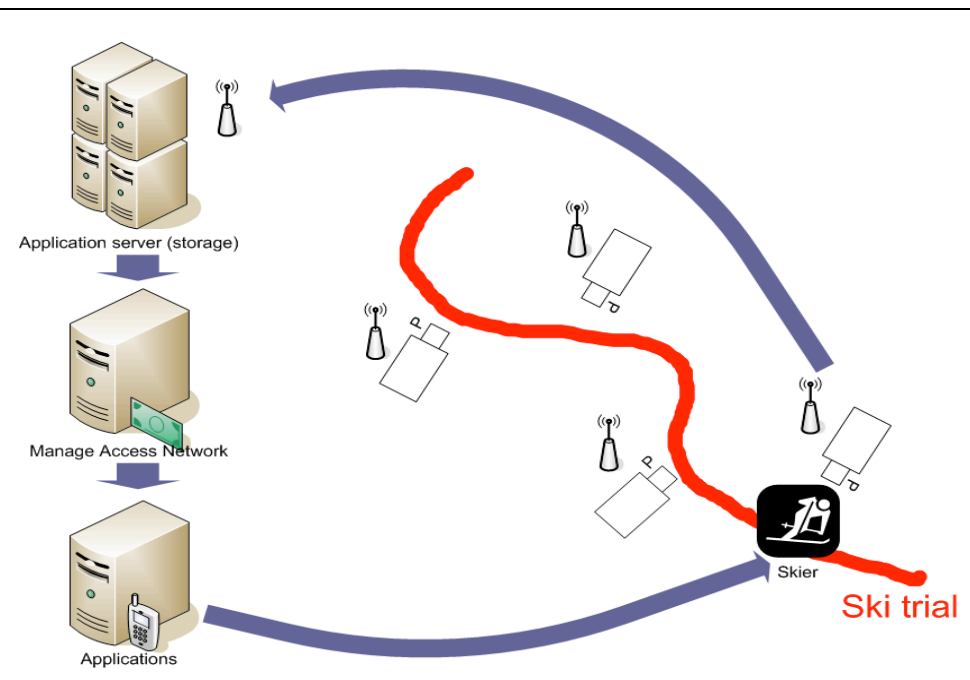

Plusieurs caméras sont installées le long d'une piste de ski et plus spécifiquement d'un snow park. Les skieurs (snowborders) sont équipés d'un capteur de mouvement, d'un téléphone mobile et d'un GPS. Les données enregistrées par les caméras sont, via un WIFI, envoyées à un serveur d'application pour stockage. Le système gère l'accès au réseau, le stockage des informations ainsi que l'envoi sur le téléphone mobile des skieurs ou sur un site Internet, des informations tels des extraits de films relatifs à des sauts ou des chutes.

Figure 3: Scénario d'application issu de l'identification des scénarios de business model

Ce nouveau scénario d'application est différent de l'idée d'usage initiale. Il est beaucoup plus restreint et se focalise sur des applications représentants des opportunités en termes de développement économique pour les opérateurs de téléphonie mobile. Partant de là, il est alors possible d'aller interroger les acteurs économiques du secteur pour mieux qualifier les scénarios obtenus. C'est la phase de qualification des scénarios que nous présentons ci-après.

\section{Partie 3 : Phase de qualification des scénarios}

L'identification de scénarios de business model est une première étape qui permet d'évaluer la cohérence et le potentiel économique du projet technologique. Ce n'est en fait que le début d'un processus qui doit conduire les acteurs économiques et les porteurs de projet de la technologie à rechercher de nouvelles informations pour affiner les business model identifiés et/ou tester leur faisabilité auprès des clients potentiels. La démarche s'enrichit donc d'une étape supplémentaire de qualification et de validation des scénarios de business model. 
Ainsi, dans le cadre du projet européen e-SENSE, le réajustement du cas d'usage a mené à des investigations supplémentaires auprès des acteurs de stations de ski. Pour cela, nous sommes allés présenter ces deux scénarios au directeur de l'office du tourisme et au directeur marketing de la société d'exploitation des remontées mécaniques d'une station de ski. Nous donnons ci-dessous un aperçu des premiers résultats, sachant qu'une étude complémentaire auprès d'autres acteurs économiques du domaine est en cours de réalisation.

Les différentes interviews réalisées ont permis de prendre en compte la logique des acteurs économiques des stations de ski.

Les acteurs de la station de ski ont réagi à nos différentes propositions. D'une part ils ont trouvé l'application séduisante pour leur clientèle et susceptible d'apporter un élément nouveau d'animation dans leur station. Cependant, ils ont d'emblé invalidé le scénario « station de ski », considérant que cela ne relevait pas de leur métier que d'investir dans une infrastructure pour proposer en direct ce type de service à leurs clients. Cette hypothèse devrait bien sur être validée par une enquête auprès d'autres stations, mais l'on peut faire l'hypothèse que le modèle d'exploitation des remontées mécaniques et les logiques de l'économie touristique sont relativement homogènes dans les différentes stations, tout du moins en France.

Nous avons donc cherché à approfondir le scénario dit « skieurs » qui consiste à proposer un service aux clients, et à leur faire payer ce service. En confrontant les hypothèses faite dans ce scénario avec l'opérateur de téléphonie mobile, nous avons pu le faire évoluer significativement, suite à nos entretiens avec les acteurs de la station de ski :

- Les clients : ce ne sont plus seulement les snow-boarders, mais aussi les familles avec enfants, et même les cyclistes (VTT ou vélo de route) qui fréquentent la station l'été

- La proposition de valeur a été resserrée autour d'une proposition simple : il s'agit de proposer aux clients de la station un dispositif permettant de filmer des événements de leur expérience dans la station : saut, leçon de ski, compétition, ou, pour les cyclistes, arrivée au sommet! Les autres éléments de la proposition de valeur comme ceux permettant d'obtenir des informations sur le trafic dans la station sont perçus comme trop compliqués.

- L'acteur central du réseau de valeur : pour les responsables de la station de ski, cela ne peut pas être l'exploitant de la station. La voie de l'opérateur peut donc être explorée, ainsi que la possibilité que cela soit un acteur qui a l'accès au marché final, comme un équipementier de sport. 
- Le modèle de paiement: nous avions imaginé dans le scénario que le client s'abonnerait au service a priori, par exemple avec l'achat de son forfait. La discussion avec les acteurs de la station nous a amené à considérer comme modèle possible l'achat des images a posteriori via une base de données accessible par Internet, en mode « pay per view ».

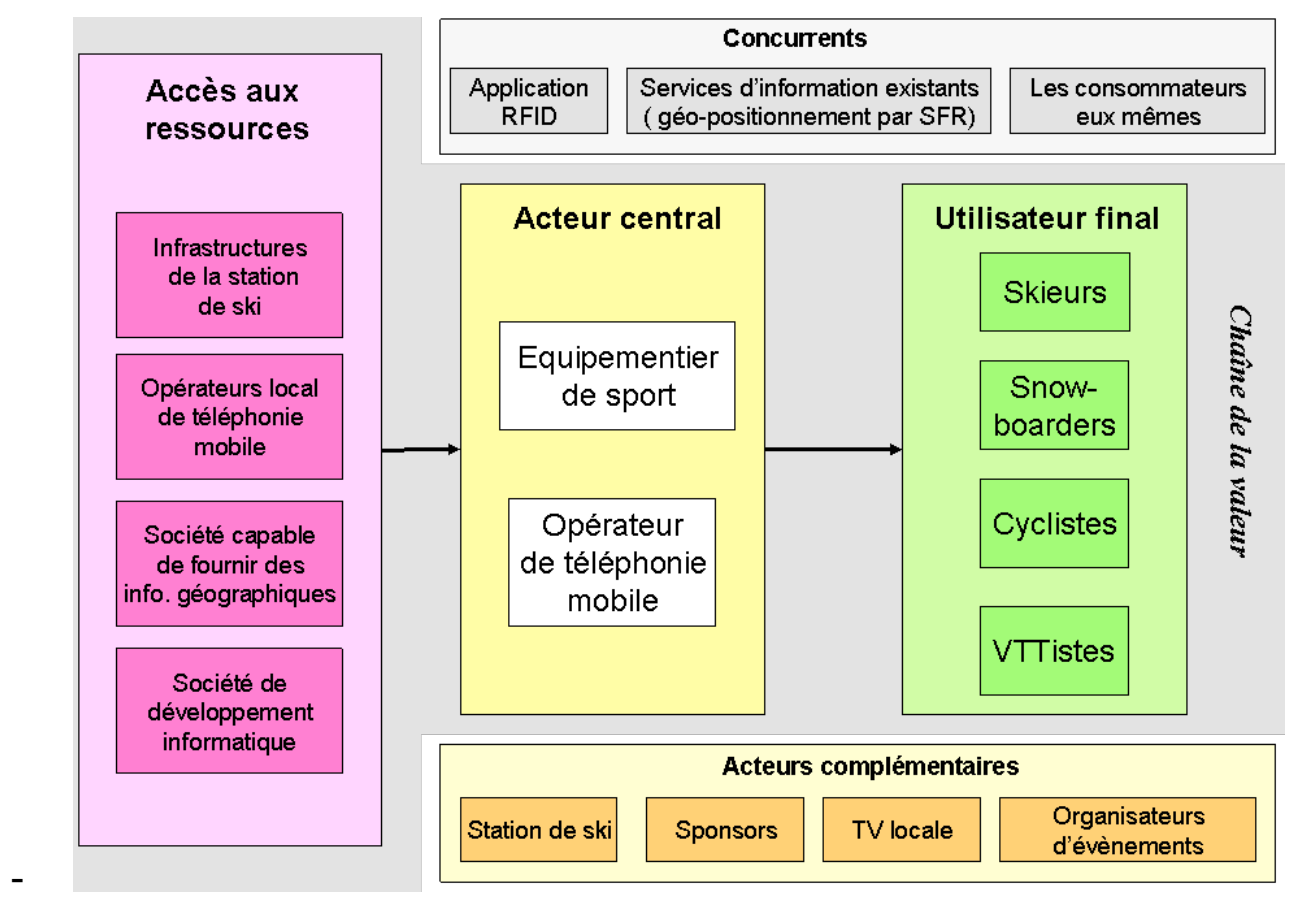

Figure 4 : Nouveau scénario de BM élaboré avec les acteurs des stations de ski et les éléments de sa chaîne de valeur

Au final, le cas d'usage identifié au début a ainsi été modifié, amélioré et surtout simplifié. Il concerne, pour la partie pratique de ski, l'ensemble des skieurs et pas uniquement les snowborders. Il peut être dupliqué à d'autres domaines d'application: le cyclisme, le VTT...et certainement à d'autres activités faites en station de ski comme la cascade de glace. L'ensemble de la démarche a fait émerger en oure td'autres acteurs autour desquels peut s'organiser l'élaboration du service.

\section{Conclusion et synthèse de la démarche suivie}

Ce travail est parti de l'hypothèse que les innovations technologiques réussies s'accompagnent souvent d'innovations dans les business models. Les managers de technologies ne sont cependant pas outillés pour réfléchir de manière structurée à la façon dont une technologie peut apporter de la valeur pour le client et selon quel modèle de création de valeur (ou business model) on peut l'amener au marché. Partant de là, cet article a présenté 
une méthode d'exploration systématique de nouveaux business models à partir d'une innovation technologique.

Le projet e-SENSE sur lequel nous avons conçu et expérimenté cette méthode de développement de scénarios de business models nous semble exemplaire des situations d'innovation technologique de type «technology push »: un projet poussé par la R\&D, de nombreuses parties prenantes (représentées ici par les partenaires industriels du consortium), une gamme d'applications possibles très large, une innovation de rupture, à la fois dans la technologie, et dans les usages.

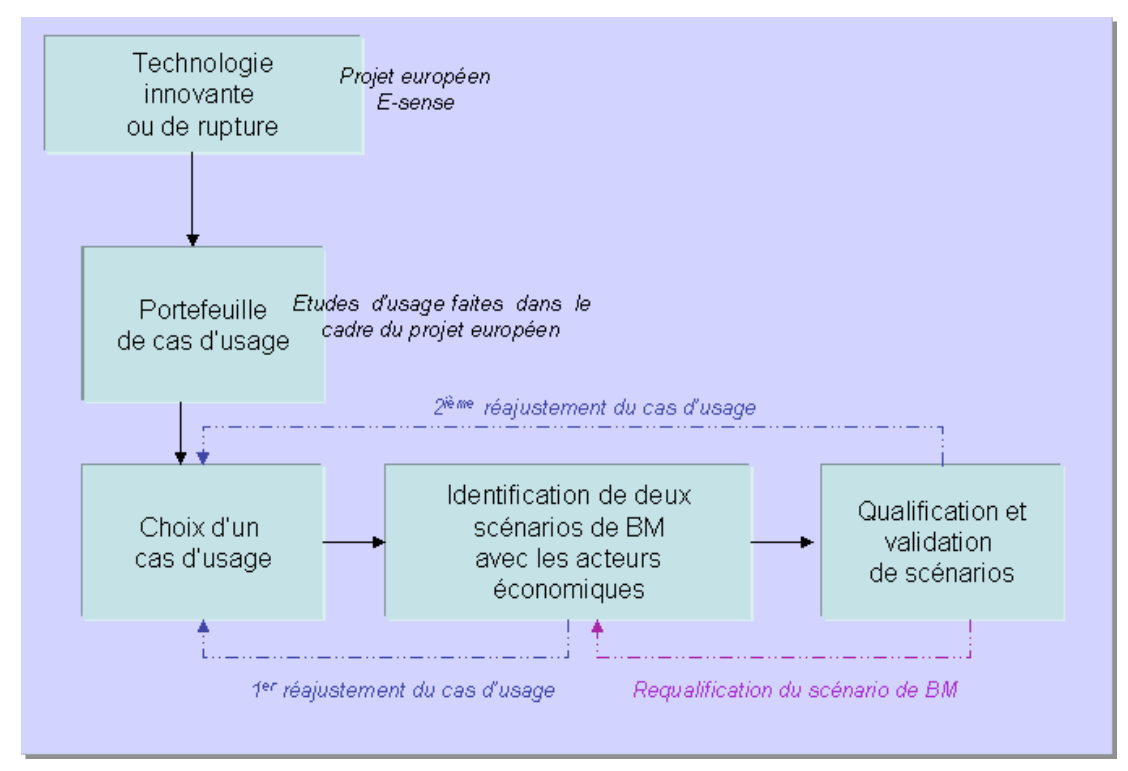

Figure 5 : Synthèse de la démarche suivie

La démarche globale suivie (cf. figure 5) montre également comment, de manière concrète, intégrer les résultats d'études anticipatives d'usage et de cas d'usages (habituellement pris en charge par des équipes marketing) et comment mettre en oeuvre une démarche de scénarios pour faciliter la délibération collective autour de nouveaux business models.

Sur la base des deux scénarios de business models, plusieurs interviews, auprès d'acteurs économiques pouvant potentiellement être intéressés par la technologie et le service associé, permettent un travail de qualification/requalification des scénarios de business model ainsi qu'une précision des cas d'usage. Nous montrons ainsi que les études socio-économiques autour des innovations technologiques se font de manière récursive, par aller-retour entre la définition des applications, la modélisation des business models, la qualification des business models, une nouvelle définition des applications et des cibles etc. Ce sont ces étapes qui accompagnent le développement technologique et permettent peu à peu d'affiner son plan de développement stratégique. 